\title{
MICROSOFT KINECT-BASED DIFFERENCES IN LOWER LIMB KINEMATICS DURING MODIFIED TIMED UP AND GO TEST PHASES BETWEEN MEN WITH AND WITHOUT PARKINSON'S DISEASE
}

\author{
Hedi KähäR ${ }^{1}$, Pille Taba ${ }^{2}$, Sven Nõmm ${ }^{3}$, Kadri Medijainen ${ }^{1}$ \\ ${ }^{1}$ Institute of Sport Sciences and Physiotherapy, University of Tartu, Tartu, Estonia \\ ${ }^{2}$ Department of Neurology and Neurosurgery, University of Tartu, Tartu, Estonia \\ ${ }^{3}$ Institute of Software Science, Tallinn University of Technology, Tallinn, Estonia
}

\begin{abstract}
The aim of the study was to analyse with Microsoft Kinect (Kinect) the differences in lower limb kinematics during sub-phases of modified Timed Up and Go test (modTUG) in men with Parkinson's disease (PD) compared to healthy age-matched male individuals. Eight men with mild-to-moderate PD (age 67.5 $\pm 4.5 \mathrm{yrs}$ ) and eight healthy men (age 69.8 \pm 8.0 yrs) participated. Kinect along with KinectPsyManager (v1.0) and Matlab2016b software was used for data collection. Selected lower limb kinematics and gait speed (GS) were calculated during sittingto-walking (STW) transition while performing modTUG. According to Kinect men with mild to moderate PD did not differ from healthy counterparts in aspects of postural characteristics of STW, with the exception of smaller distance between knees while sitting $(\mathrm{p}<0.001)$. Men with PD were found to perform the walking phase of STW transition slower $(\mathrm{p}<0.01)$ and with slower GS $(\mathrm{p}<0.01)$ comparing to healthy men. In conclusion, compared to healthy men, Kinect detects smaller distance between knees during sitting before transitioning from STW in men with mild to moderate PD. In addition, men with PD also demonstrated slower GS and a longer walking phase of STW transition in comparison to healthy men.
\end{abstract}

Keywords: Microsoft Kinect; Parkinson's disease; modified TUG test; sittingto-walking 


\section{INTRODUCTION}

Parkinson disease (PD) is one of the most common neurodegenerative diseases among elderly. The occurrence of PD rises with age, with an age-specific prevalence of 425 per 100,000 at the age group of 65-74, and 1903 per 100,000 in individuals over 80 yrs [19]. The cause of the disease still remains unknown and is often classified as idiopathic or sporadic PD $[5,11]$.

The mechanisms of PD are complex. The main pathological marker for $\mathrm{PD}$ is considered to be the degeneration of the cells in basal ganglia, which leads to insufficient levels of dopamine, resulting in motor symptoms of the disease [12]. The characteristic features of PD include motor symptoms such as resting tremor, rigidity, bradykinesia and postural instability [12].

Due to the motor symptoms, everyday tasks involving transfers, gait and gait-related activities are affected in PD. One of the activities many individuals with PD struggle with is standing up from a chair and walking [23]. STW is a frequently required movement of everyday life [23] and a basic transfer needed for mobility and functional independence [11].

As PD is a neurodegenerative disease, the condition of the patient pursues to worsen over time [12]. It is crucial to continue monitoring the progression of the disease and functionality of the patient in order to offer the best possible support and care for people living with PD.

There are a number of assessment measures available to assess the functionality and mobility. Timed Up and Go test (TUG) is one that can be applied in PD population [21]. TUG has been used to assess patients with moderate to severe PD [22].

TUG is a widely used functional test to assess and predict falls, declines in health and difficulties with activities of daily living in elderly [16, 24]. A classical TUG test assesses the patient's ability to perform sequential locomotors tasks of standing up, walking $3 \mathrm{~m}$ turning and walking back to sit down. The standard procedure of TUG test measures the duration (in seconds) of the aforementioned performance [9]. However, recent findings report that in assessing patients with early to mild stage of $\mathrm{PD}$, the classical TUG test is not sensitive enough to detect abnormalities [22].

In recent years there have been an increasing number of studies where different type of motion sensors have been used along with standard performance of TUG. Studies have revealed that instrumented TUG (iTUG) can provide more information comparing to measures taken by a classical TUG $[15,21,22]$. A major part of this research includes use of different types of camera based assessment tools for instrumental motion analysis $[4,6]$.

Conventional video-based assessment tools like 3D gait analysis systems (e.g. Vicon) are the gold standard for analysis of gait and other motions. The drawback of Vicon-like systems is the need for spacious (laboratory) 
settings, usage of reflective markers, many cameras and competent experts. Altogether, this assessment method is very resource-dependant [6].

A rather new, simple and low-cost motion sensor is found to be the Kinect sensor by Microsoft Inc. Kinect is a camera-based sensor mostly known as an accessory for a gaming console known as Xbox. Kinect recognises movement of the body without additional body markers or force platforms, and has proven to be reliable in measuring temporal characteristics of people with $\mathrm{PD}[6]$.

Combining a standard outcome measure like TUG test along with Kinect can provide extended information. Recording spatiotemporal characteristics of different phases of a functional activity (e.g. getting up from a chair and walking) potentially enables the development of specific and targeted interventions that addressees the motor limitations contributing to the impaired performance. Later is beneficial for increasing or maintaining the functionality and independence of a person living with PD.

The purpose of the current study was to analyse differences of lower limb kinematics and temporal characteristics detectable by Kinect during STW sub-phase of modTUG test in male individuals with PD compared to healthy male counterparts.

\section{MATERIALS AND METHODS}

\section{Subjects}

The study was conducted as a part of a larger project "Changes of functional capacity among patients with Parkinson's disease" and has been approved by the Ethics Committee of the University of Tartu (Certificate nr 245/M-25, dated 16.02.2015).

Sixteen men, recruited from The PD Association of Tartu and Tallinn, were divided into two equal sized and age-matched groups - PD-group and Control group (CG). All the PD-group participants had confirmed PD diagnosis (according to the Queen Square Brain Bank (QSBB) criteria) by an expert neurologist and were examined during "on"-period (in a case of existing motor fluctuations as a complication of levodopa therapy) [20]. CG consisted of age-matched healthy elderly men. The exclusion criteria included previous cardio-vascular incident, concurrent neurological condition, or presence of any other untreated medical condition that might affect mobility. In addition, participants with moderate to severe cognitive impairment on Mini Mental State Examination and persons who used walking aids were excluded from the study. The PD and CG did not differ in means of demographic parameters (Table 1). 
Table 1. Participants demographic characteristics (mean \pm standard deviation).

\begin{tabular}{lcc}
\hline & PD group $(\mathbf{n}=8)$ & CG group $(\mathbf{n}=8)$ \\
\hline Age $(\mathrm{yrs})$ & $67.5 \pm 4.5$ & $69.8 \pm 8.0$ \\
\hline Height $(\mathrm{m})$ & $1.8 \pm 0.1$ & $1.7 \pm 0.1$ \\
\hline Weight $(\mathrm{kg})$ & $91.3 \pm 10.5$ & $90.0 \pm 18.8$ \\
\hline BMI $\left(\mathrm{kg} / \mathrm{m}^{2}\right)$ & $29.4 \pm 3.0$ & $30.7 \pm 6.0$ \\
\hline
\end{tabular}

PD - Parkinson's disease, CG - Control, $\mathrm{n}$ - number of participants in the group, BMI Body Mass Index.

\section{Procedure}

ModTUG test was used for data collection to analyse the differences detectable with Kinect in lower limb kinematics of STW performance while performing modTUG test. Kinect was used for this study in order to track and save the movements of hip and knee joints.

Kinect is a camera based gadget that uses infra-red sensors and colour sensors to track the movements of subjects. It has a built-in software that allows to detect 20 points of the body (large body joints) thereby constructing a digital image of the body. The use of additional body markers is not needed [13].

The software KinectPsyManager v1.0 was developed for Kinect in the Tallinn University of Technology. The software enabled the recording the movements of participants in different planes. Matlab2016b software was used to transform the collected data the recorded movement coordinates into joint angles of interest. In current study, hip and knee joints were chosen for data analysis.

ModTUG test was used due to restrictions by Kinect sensor sensitivity: there is an optimal distance where Kinect can accurately detect movements [13]. Based on previous research using Kinect, it was found that the maximal walking distance that would still enable to obtain accurate data would be $2.08 \mathrm{~m}$. Therefore, the participants performed TUG test according to standard protocol with the exception of shorter walking distance instead to the standard $3 \mathrm{~m}$. Kinect was placed at a height of $90 \mathrm{~cm}, 3.35 \mathrm{~m}$ away from the chair, facing the participant.

Participants were instructed to stand up, walk to the cone placed 2.08 $\mathrm{m}$ from the chair, turn around the cone over dominant shoulder and walk to the chair and return to sitting (with back supported). Three consecutive trials were performed. Five "Markers" were distinguished during execution of the modTUG: 
- Marker 1: the participant is seated;

- Marker 2: the participant is standing (maximal extension in both hip and knee joints) position;

- Marker 3 the participant has walked to the cone and starts the turn;

- Marker 4 the participant has ended the turn;

- Marker 5 the participant has walked back to the chair (and is facing it);

- Marker 1 (start of new attempt) the participant has sat back down (back against the chair).

All the markers were manually adjustable during data analysis process. In the current study, Markers 1-3 were chosen as the main focus was to analyse the STW performance. STW was further divided into two phases: sit-tostand (STS) (Marker 1 to Marker 2) and walking (Marker 2 to Marker 3).

Postural characteristics of STW were derived from data recorded by Kinect: Maximal joint angles (in degrees) were used to clarify if there is a deficiency in extension when standing, as PD patients are known to be characterised by stooped posture [5]. In addition, the minimal joint angles were included to enable the calculation of the range of motion (ROMcalculated as a subtraction of minimal joint angle from maximal joint angle, measured in degrees) demonstrated by the participant during total motion of STW captured by Kinect. ROM is often reduced in PD due to rigidity and bradykinesia [5].

The distance between knees during STW motion was also recorded as another postural parameter of interest to characterise the step width and base of support of the participants. The maximal and minimal distances between knees (in $\mathrm{cm}$ ) were recorded for STW and sub-phases.

The duration of STW and sub-phases (STS and walking) was recorded. Additionally, GS was calculated (m/s).

The mean of three trials was used for data analysis to compare men with $\mathrm{PD}$ and the CG.

\section{Statistical analysis}

Statistical analysis was performed with two commercially available statistical softwares. Descriptive analysis (mean \pm standard deviation) was performed using Microsoft Excel (2016) and further statistical analysis was conducted with R-studio software. The distribution of data was analysed with Shapiro-Wilk test. Differences between groups were analysed depending on the distribution of data using either student-t or Wilcoxon test. The level of significance was set as $\mathrm{p}<0.05$. 


\section{RESULTS}

\section{Postural characteristics}

It was found that based on recordings by Kinect both participants in PD group and CG reached similar, near fully-extended positions in both hip and knee joints while performing STW phase of modTUG test. No differences in joint excursion were found (Table 2). As no differences between right and left side values of the registered parameters were found in either group, the results are presented as mean values of the right lower limb.

Table 2. Maximal joint angles and range of motion while performing sitting-to-walking measured with Kinect in degrees (mean \pm standard deviation).

\begin{tabular}{lcc}
\hline & PD group $(\mathbf{n}=\mathbf{8})$ & CG $(\mathbf{n}=\mathbf{8})$ \\
\hline RHJ max & $175.0 \pm 3.3$ & $175.0 \pm 3.3$ \\
\hline RKJ max & $179.0 \pm 0.6$ & $178.7 \pm 1.2$ \\
\hline RHJ ROM & $59.8 \pm 8$ & $57.8 \pm 8.2$ \\
\hline RKJ ROM & $64.5 \pm 8.4$ & $64.5 \pm 8$ \\
\hline
\end{tabular}

PD - Parkinson's disease, CG - control group, ROM - range of motion, $\mathrm{RHJ}$ - right hip joint, RKJ - right knee joint.

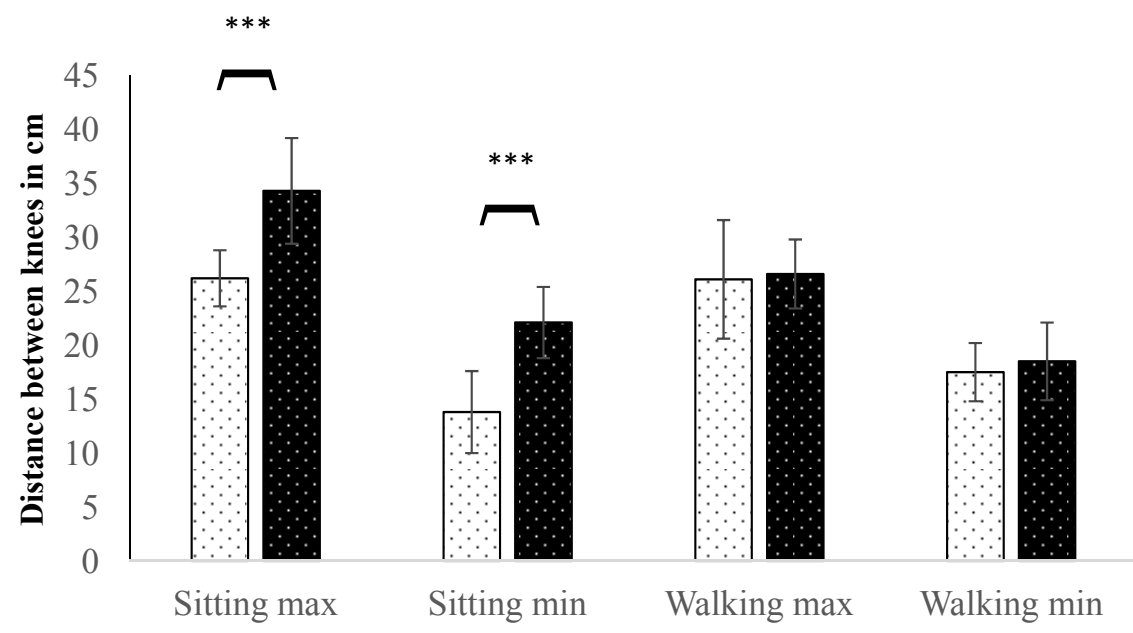

$\square \mathrm{PD} \cdot \mathrm{CG}$

Figure 1. Maximal (max) and minimal ( $\mathrm{min})$ distance between knees in centimetres $(\mathrm{cm})$ during sitting and walking phase of sitting-to-walking. Parkinson's disease (PD) and Control group (CG), ${ }^{* * *} p<0.001$ 
The distance between knees differed in men with and without PD during STW performance. The difference arises from sitting position, where the maximal and minimal distance between knees was significantly reduced $(\mathrm{p}<0.001)$ in men with PD compared to CG. The results are illustrated in Figure 1.

\section{Temporal characteristics}

The duration of STW was found to be significantly longer $(\mathrm{p}<0.01)$ in PD patients compared to healthy elderly ( $4.2 \pm 1 \mathrm{sec}$ vs $3 \pm 0.5 \mathrm{sec}$, respectively) to stand up and walk $2.08 \mathrm{~m}$ than people in CG. A more detailed look revealed that total duration was increased due to slower walking phase of the STW performance. At the same time, no difference in STS was found (see Figure 2). PD patients walked significantly slower $(\mathrm{p}<0.001)$. $(0.77 \pm 0.2 \mathrm{~m} / \mathrm{s}$ in $P D$ vs $1.08 \pm 0.2 \mathrm{~m} / \mathrm{s}$ in CG, respectively).

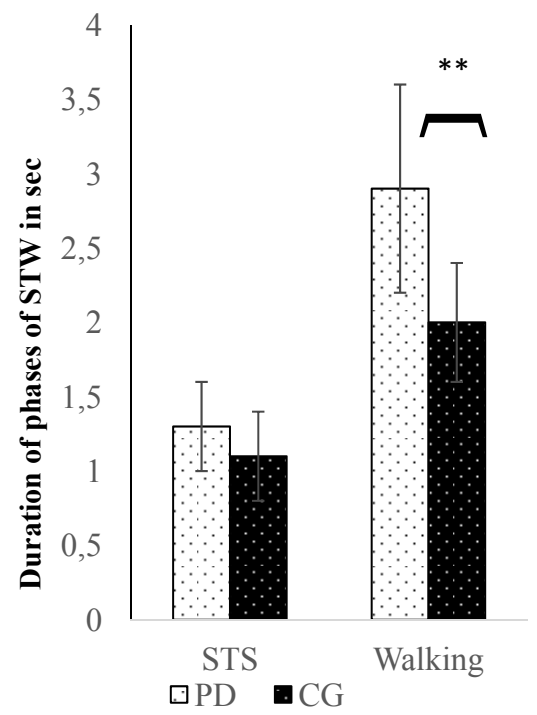

Figure 2. Sitting-to-walking (STW) motion in two phases: sit-to-stand (STS) and walking, between Parkinson's disease (PD) and Control group (CG) ${ }^{* *} p<0.01$ in seconds (sec)

\section{DISCUSSION}

The aim of this study was to analyse the differences of selected kinematic characteristics detectable by Kinect during specific phases of modTUG test in male individuals with Parkinson's disease compared to healthy agematched men. 
Though, due to presence of bradykinesia in PD patients, it could be expected to find reduced movement amplitude in individuals with PD [5]. However, this was not the case in current study neither in the extent of extension in lower limbs nor in joint excursion demonstrated during STW.

Both men with and without PD reached near-full extension in hip and knee joints during STW. Nugis [17] reported similar results in a study comparing the performance of Five-Times STS test in PD and healthy elderly. Our findings are also in accordance with Cachia [2] who established similar findings when comparing PD participants' lower limb joint angles to healthy elderly while performing STS.

In the current study, no differences were found between groups in the ROM of knee and hip joints during STW. An assessment of straight line walking of patients with PD similarly did not detect any differences in ROM of knee and hip joints [4]. A previous study comparing STW in PD patients and healthy counterparts during performance of iTUG found no differences in trunk flexion range [23].

A possible explanation for not finding any differences between PD patients and CG in joint angles in our current study could probably lie in stage of PD. Changes in posture that may be associated with rigidity, like increased flexion and reduced movement amplitude in neck, trunk, upper and lower limbs usually develop in the later stages of the disease [10]. The participants of the current study had mild- to- moderate PD. It is possible that the results would have revealed differences between groups if the patients with moderate-to-severe PD were examined.

Moreover, PD participants were assessed while being in "on"-period with motor symptoms better controlled, allowing participants with PD to move at their best possible level [20]. It is likely that when the study would have been conducted with the participants being in "off"-period, the differences in postural characteristics could have been evident between groups.

Nonetheless, despite no differences were found in our study, it is important to assess ROM and also to educate patients to maintain it in order to prevent loss in $\mathrm{ROM}$ in the future as during the disease progression, also rigidity may worsen $[5,10]$.

In the current study, the distance between knees was measured during STW movement. Measuring the distance between knees can reflect information about step width and base of support. In current study, PD participants did not reach the same maximal distance between knees while performing STW. When looking at the separate parts of the movement- sitting and walking, no differences in distances between knees were found during walking. These findings are supported by previous studies, where also no 
difference in step width during walking was found between PD and CG groups $[1,4]$.

As mentioned above, the difference found between groups resulted from sitting position, where the distance between knees was smaller in PD participants. Taking into account the previously established findings that an optimal base of support is needed for safe STS movement to decrease risk of falling [11], the results show that participants with PD in current study potentially are not using an optimal base of support and could be at higher risk of falling due to that while performing STS movement.

The reason behind differences in knee distances among the two groups might be due to muscle strength. It is known that PD participants are prone to have weakness in muscles surrounding hip and knee joints [14] which would make it more difficult to maintain a stable distance between knees. This should be considered when planning therapy: targeted muscle strengthening to hip abductors and adductors and practising optimal base of support during transfers could help to decrease the risk of falling in people with PD.

Chen [3] established that compared to non-fallers, elderly who had recurrent falls, performed the STW movement of TUG test slower. 70-87\% of people with PD will fall at one point during their disease [8]. In current study PD patients also performed STW movement of modTUG test slower compared to $C G(p<0.01)$. These findings are similar with a study by Van Uem [23] where PD participants also performed STW part of iTUG significantly slower.

A more detailed look into STW duration revealed that the duration of STW was mainly increased due to longer duration of walking phase in PD group. This is in accordance with findings from Eltoukhy [4] who found similarly that PD participants are slower in walking tasks compared to healthy elderly.

The duration of STS phase of STW was similar in PD and CG group. These findings are in agreement with a previous study where the STS transfer during Five-Times STS test was similar in PD patients compared to healthy elderly [17].

Previous research has established that patients with PD have slower GS when compared to healthy elderly [5], and our results further support this. However, the GS of PD patients in the current study was considerably slower compared to a study of Parker [18] $(0.77 \mathrm{~m} / \mathrm{s}$ vs $0.94 \mathrm{~m} / \mathrm{s})$, though the stage of the disease was similar in both of the studies. Findings of the current study are closer to average GS of $0.88 \mathrm{~m} / \mathrm{s}$ detected in PD participants with more advanced disease (H\&Y score 3-4) [7]. 
However, at least partly the slow GS of our PD participants can be attributed to the fact that GS was recorded during functional activity of STW performed on a short walking trail. Besides the short distance also acceleration (post standing up and starting to walk) and deceleration (prior to turning) were included in our study. In contrast, Hass [7] and Parker [18] measured the GS while straight line walking. However, as CG performed under same circumstances, the difference in GS between PD patients and controls is evident and the slowness of PD patients cannot be solely attributed to the short walking distance. The slower GS of PD participants might potentially arise from other PD characteristics symptoms like bradykinesia or start hesitation [5].

Another reason for slow GS could be the use of a non-specific room. GS is often measured in gait laboratories [18]. Zampieri [22] found that PD patients walk slower in home settings compared to gait laboratories settings while performing iTUG test. The room used in our study was comparable to home-environments due to its size.

We recognize there are some limitations of our study. The main limitation of the study was the relatively small sample size. Additionally, the usage of Kinect to assess PD patients with more advanced disease is limited, as currently the system does not enable the detection and recording of motion in case of more than one individual in the vision range of the system. However, patients with severe $\mathrm{PD}$ would definitely require someone to safeguard their performance. In addition, the possibility to record postural and temporal characteristics while turning would provide valuable additional information.

Future studies should concentrate on the turning phase and stand-to-sitphase of TUG test. Additionally, a similar study comparing PD participants with and without history of falls (and fear of falling), could increase the understanding about the associations between performance of functional activity such as STW and falls risk of PD patients.

The main strength of the current study was the use of a relatively new method Kinect for analysis of a classical functional test like modTUG for data collection. We used a cost- effective alternative to the gold standard of 3D motion analysis systems that can be used in more flexible environments resembling home and clinical settings [22,23]. Additionally, the possibility to analyse with Kinect and objectively assess the temporal characteristics of specific phases of STW provides extended information compared to standard assessment of TUG. 


\section{ACKNOWLEDGEMENTS}

We would like to thank all the participants, their families and the PD Associations of Tartu and Tallinn for their part in the project. Additionally, we wish to express our gratitude to Eveli Tamm, Dan Uvarov, Marita Nugis and Martti Vaske for their contribution in the data collection process. This study was supported by the Grants GMVCM1239P and IUT2-4 of the Estonian Research Council.

\section{REFERENCES}

1. Bovonsunthonchai S, Vachalathiti R, Pisarnpong A, Khobhun F, Hiengkaew. (2014) Spatiotemporal gait parameters for patients with Parkinson's Disease compared with normal individuals. Phys Ther Res Int, 19: 158-165.

2. Cachia C. (2008) A biomechanical analysis of the sit-to-stand transfer in Parkinson's disease. Master's thesis. Kingston, Kanada: Queen's University.

3. Chen T, Chang CC, Chou L-S. (2013) Sagittal plane centre of mass movement strategy and joint kinetics during sit-to-walk in elderly fallers. Clin Biomech, 28: 807-812.

4. Eltoukhy M, Kuenze C, Oh J, Jacopetti M, Wooten S, Signorile J. (2017) Microsoft Kinect can distinguish differences in over-ground gait between older persons with and without Parkinson's disease. Med Eng Phys, 44: 1-7.

5. Falup-Pecurariu C, Ferreira J, Martinez-Martin P, Chaudhuri KR. (2017) Movement disorder curricula. Wien: Springer-Verlag, 29-43: 95-117.

6. Galna B, Barry G, Jackson D, Mhiripiri D, Olivier P, Rochester L. (2014) Accuracy of the Microsoft Kinect sensor for measuring movement in people with Parkinson's disease. Gait Post, 39: 1062-1068.

7. Hass CJ, Malczak P, Nocera J, Stegemoller EL, Wagle SA, Malaty I, Jacobson IV CE, Okun SM, McFarland N. (2012) Quantitative normative gait data in a large cohort of ambulatory persons with Parkinson's disease. PLoS ONE, 7: e42337.

8. Hely MA, Reid WG, Adena MA, Halliday GM, Morris JG. (2008) The Sydney multicenter study of Parkinson's disease: the inevitability of dementia at 20 years. Mov Disord, 23: 837-844.

9. Herman T, Giladi N, Hausdorff JM. (2011) Properties of the timed up and go test: more than meets the eye. Gerontol, 57: 203-210.

10. Jankovic J. Parkinson's disease: clinical features and diagnosis. (2008) J Neurol Neurosurg Psychiatry, 79: 368-376.

11. Janssen, WGM, Bussmann, HBJ, Stam, HJ. (2002) Determinants of the Sit-toStand Movement: A Review. Phys Ther, 82: 866-879.

12. Kalia LV, Lang AE. (2015) Parkinsons's disease. Lancet Neurol, 386: 896-912

13. Lissenko H. (2015) Using multiple Kinect sensors to register turning movement. Master thesis. Tallinn. Tallinna Tehnikülikool [in Estonian]. 
14. Mak MKY, Hui-Chan CMY. (2004) Audiovisual cues can enhance sit-to-stand in patients with Parkinson's disease. Mov Disord, 19: 1012-1019.

15. Mellone S, Tacconi C, Chiari L. (2012) Validity of a smartphone-based instrumented Timed Up and Go. Gait Post, 36:163-165.

16. Nocera JR, Stegemöller EL, Malaty IA, Okun MS, Marsiske M. (2013) Using the Timed Up \& Go test in a clinical setting to predict falling in Parkinson's Disease. Arch Phys Med Rehabil, 94: 1300-1305.

17. Nugis M. (2016) Customly and with Microsoft Kinect assessed five times sit-tostand test in men and women with Parkinson's disease before and after single physical therapy intervention. Master's thesis. Tartu, Estonia: University of Tartu [in Estonian].

18. Parker N, Bugdayci D, Goksenoglu G, Demircioglu DT, Kesiktas N. (2015) Gait speed and related factors in Parkinson's disease. J Phys Therap Sci, 27: 3675-3679.

19. Pringsheim T, Jette N, Frolkis A, Thomas, Steeves DL. (2014) The prevalence of Parkinson's Disease: A systematic review and meta-analysis. Mov Disord, 29: $1583-1590$.

20. Rascol O. (2016) Pharmacological insights into Levodopa-induced motor fluctuations in patients with Parkinson's Disease. Mov Disord Clin Pract, 3: 523-526.

21. Shulman LM, Armstrong M, Ellis T, Gruber-Baldini A, Horak F Nieuwboer A, Parashos S, Post B, Rogers M, Siderowf A, Goetz CG, Schrag A, Stebbins GT, Martinez-Martin P. (2016) Disability Rating Scales in Parkinson's Disease: Critique and Recommendations. Mov Disord, 31: 1455-1465.

22. Zampieri C, Salarian A,Carlson-Kuhta P, Nutt JG, Horak BF. (2011) Assessing mobility at home in people with early Parkinson's disease using an instrumented Timed Up and Go test. Park Rel Dis, 12: 277-280.

23. Van Uem JMT, Walgaard S, Ainsworth E, Hasmann SE, Heger T, Nussbaum S, Hobert MA, Mico-Amigo EM, Van Lummel RC, Berg D, Maetzler W. (2012) Quantitative Timed-Up-and-Go parameters in relation to cognitive parameters and health-related quality of life in mild-to-moderate Parkinson's Disease. PLoS ONE 11(4):1-15.

24. Viccaro LJ, Perera S, Studenski SA. (2011) Is Timed Up and Go better than gait speed in predicting health, function, and falls in older adults? J Am Geriatr Soc, 59: 887-892.

\section{Correspondence to:}

Hedi Kähär

Institute of Sport Sciences and Physiotherapy

University of Tartu

Tartu, Estonia

E-mail: hedikahar@gmail.com 